\title{
Comparative Study of Clinical and Radiographic Outcomes of High Tibial Osteotomy in Patients with Kissing Lesions and Non-Kissing Lesions
}

\author{
Oog-Jin Shon, MD, Sang-Jin Park, MD, Bum-Jin Shim, MD, and Dong-Yeol Lee, MD \\ Department of Orthopedic Surgery, Yeungnam University Medical Center, Daegu, Korea
}

\begin{abstract}
Purpose: High Outerbridge grade lesions of the articular cartilage have been associated with poor outcomes of high tibial osteotomy (HTO). However, there has been no report on the efficacy of HTO in the presence of kissing lesions. The purpose of this study was to compare clinical and radiographic outcomes of HTO between kissing lesion and non-kissing lesion groups.

Materials and Methods: Of the patients with medial compartment osteoarthritis and varus deformity treated with HTO between 2007 and 2012,21 cases with kissing lesions and 22 cases without kissing lesions were selected. Clinical outcomes were assessed using the Lysholm knee scoring scale score, visual analogue scale score, Hospital for Special Surgery score, Western Ontario and McMaster Universities score, and Tegner activity score. Radiographic outcomes were assessed using the femoral-tibial angle, mechanical axis deviation, medial proximal tibial angle, posterior tibial slope angle, and joint space width.

Results: Clinical outcomes were improved in both groups from the postoperative assessment to the final follow-up, which showed no statistically significant difference between groups at the final follow-up. No statistically significant differences were observed with regard to radiographic assessment. Conclusions: Since both groups showed favorable short-term clinical and radiographic outcomes, HTO might be an alternative treatment option for degenerative osteoarthritis with kissing lesions in relatively young patients.
\end{abstract}

Keywords: Knee, Osteoarthritis, Osteotomy, Kissing lesion

\section{Introduction}

Unicompartmental arthritis of the knee can be treated with surgical methods such as high tibial osteotomy (HTO) and unicompartmental knee arthroplasty (UKA). It is important to decide on an appropriate method for the patient ${ }^{1,2)}$. Many researchers have reported high survival rates of HTO. Insall et al. ${ }^{3)}$ reported that it resulted in a 5- and 9-year survival rate of $85 \%$ and $63 \%$, respec-

Received September 30, 2016; Revised (1st) December 5, 2016;

(2nd) December 8, 2016; (3rd) January 26, 2017;

(4th) March 9, 2017; Accepted April 7, 2017

Correspondence to: Bum-Jin Shim, MD

Department of Orthopedic Surgery, Yeungnam University Medical

Center, 170 Hyeonchung-ro, Nam-gu, Daegu 42415, Korea

Tel: +82-53-620-3640, Fax: +82-53-628-4020

E-mail: redpross@naver.com

This is an Open Access article distributed under the terms of the Creative Commons Attribution Non-Commercial License (http://creativecommons.org/licenses/by-nc/4.0/) which permits unrestricted non-commercial use, distribution, and reproduction in any medium, provided the original work is properly cited. tively, and Naudie et al. ${ }^{4}$ reported its 5- and 9-year survival rate as $95 \%$ and $80 \%$, respectively. There is consensus that HTO can be an ideal surgical option for active patients aged $\leq 65$ who have a degenerative lesion with around $10^{\circ}$ moderate varus or valgus deformity limited to a lateral or medial compartment and have $\geq 90^{\circ}$ flexion and $\leq 15^{\circ}$ flexion contracture ${ }^{4-6)}$. However, HTO is relatively contraindicated for unstable knee joint with ligament loosening, severe degenerative changes of the patellofemoral joint, obese patients, advanced age population, or less active patients. UKA is preferred in those cases ${ }^{7,8)}$.

HTO is also known to be a relative contradiction for advanced arthritis with a relatively moderate degenerative change of the patellofemoral joint ${ }^{9)}$ accompanied by kissing lesion ${ }^{10,11)}$, and UKA has been preferred instead in such a case. In young patients, however, UKA or total knee arthroplasty (TKA) can be less useful because of the risk for revision arthroplasty, extended life expectancy and various complications such as limited activity $^{12,13)}$. There are a few reports in the literature describing HTO combined with arthroscopic microfracture in young arthritis 
patients with Outerbridge grade III and IV chondropathy on the femoral and tibial joint surface, which we think could be diagnosed as kissing lesions ${ }^{14,15}$. They suggested that this type of treatment was clinically and radiographically more effective in getting rid of pain, preserving joints, and performing daily living activities than UKA in a short-term follow-up. However, to the best our knowledge, there has been no report comparing clinical and radiographic outcomes of HTO between patients with kissing lesions and non-kissing lesions.

The purpose of this study was to compare clinical and radiographic outcomes of HTO between kissing lesion and nonkissing lesion groups involving relatively young patients with mild medial meniscus damage that can be treated with partial meniscectomy or suture.

\section{Materials and Methods}

\section{Patient Demographics}

This study was approved by the Institutional Review Board of our hospital, and informed consent was obtained from all participants. We reviewed 50 patients who underwent HTO combined with arthroscopic microfracture to treat medial compartment arthritis accompanied by mild varus deformity (femoral-tibial angle $[\mathrm{FTA}]>1^{\circ}$ ) and mild to moderate degenerative arthritis (Kellgren-Lawrence grade ${ }^{16)} 1$ to 3 ) in our hospital from May 2007 to May 2012. Seven patients were excluded due to less than 2 years of follow-up (4 patients), lesions in the lateral meniscus or lateral compartment arthritis (3 patients). The remaining 43 patients were divided into the kissing group (group $\mathrm{I}, \mathrm{n}=21$ ) and non-kissing group (group II, $\mathrm{n}=22$ ) (Table 1) in order to perform comparative analysis. The mean age was 57.4 years in group I and 55.5 years in group II. The mean duration of follow-up was 34.5 months (range, 24 to 52 months).

Table 1. Patient Demographics

\begin{tabular}{lccc}
\hline \multirow{2}{*}{ Variable } & $\begin{array}{c}\text { Kissing lesion } \\
\text { group }(\mathrm{n}=21)\end{array}$ & $\begin{array}{c}\text { Non-kissing lesion } \\
\text { group }(\mathrm{n}=22)\end{array}$ & p-value \\
\hline Age (yr) & $57.4(44-63)$ & $55.5(41-64)$ & $0.301^{\mathrm{a})}$ \\
Sex (M:F) & $2: 19$ & $7: 13$ & $0.067^{\mathrm{b})}$ \\
femoral-tibial angle $\left(^{\circ}\right)$ & Varus 4.6 (1-10) & Varus 3.5 (1-7) & $0.242^{\mathrm{a})}$ \\
BMI $\left(\mathrm{kg} / \mathrm{m}^{2}\right)$ & $27.6(20.3-29.1)$ & $25.0(20.2-31.1)$ & $0.100^{\mathrm{a})}$ \\
Follow-up period $(\mathrm{mo})$ & $30.0(24-49)$ & $30.5(26-52)$ & $0.637^{\mathrm{c}}$ \\
\hline
\end{tabular}

Values are presented as mean (range).

${ }^{\text {a) }}$ Student $t$-test.

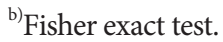

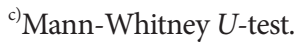

The kissing lesion affects both the contacting femoral and tibial condyles. It was diagnosed when the femoral condyle and tibial condyle were in contact with each other on weight bearing posteroanterior radiograph taken at $45^{\circ}$ of knee flexion. Medial meniscus repair was done in 3 patients (14\%) in group I, and 4 patients (19\%) in group II. Partial meniscectomy was done in 4 patients (18\%) in group I, and 3 patients (14\%) in group II. Other patients underwent simple cartilage debridement.

\section{Operative Technique}

The surgical indication was medial knee arthritis accompanied by mild varus deformity found in weight bearing radiographic evaluation. In addition, we selected patients who had no osteophyte in the lateral compartment, who had reasonable joint space preservation and who had medial meniscus damage alone. Arthroscopy with microfracture was performed in each patient preoperatively and partial meniscectomy or suture was performed when medial meniscus damage was found. Surgeon pulled the torn meniscus with an arthroscopic probe and decided partial meniscectomy or suture.

A longitudinal skin incision was made on the medial side of the tibial tuberosity. The superficial medial collateral ligament was completely released below the osteotomy site. Biplanar medial open wedge osteotomy was performed using chronOS vivify spacer (DePuy Synthes, Solothurn, Switzerland) and TomoFix plate (DePuy Synthes). Target correction angle was measured at the point where the mechanical axis of the lower limb passed through the Fugisawa point, which is $62.5 \%$ from the medial tibial articular margin.

\section{Rehabilitation Protocol}

We used a venous compression pump intermittently along with an ice bag postoperatively. The day after the operation, the patients were allowed to do joint movement in a knee brace and began to do quadriceps strengthening exercise. A continuous passive motion machine was used from the 3 rd postoperative day for about 8 weeks. Partial weight bearing and crutch gait were allowed according to radiographic findings at the osteotomy site eight weeks postoperatively. Full weight bearing was initiated after bone union was confirmed at the osteotomy site in the radiographic evaluation 12 weeks postoperatively.

\section{Evaluation (Clinical and Radiographic Outcome and Second Look Arthroscopic Findings)}

Clinically, authors evaluated the Lysholm knee scoring scale score $^{17)}$, visual analogue scale (VAS) score ${ }^{18)}$, Hospital for Special 
Surgery (HSS) score ${ }^{19)}$, Western Ontario and McMaster Universities (WOMAC) score ${ }^{20)}$, and Tegner activity score ${ }^{21)}$ preoperatively, 3, 6, and 12 months postoperatively and at the final followup.

Radiographically, authors measured the FTA, mechanical axis deviation (MAD), medial proximal tibial angle (mPTA), posterior tibial slope angle (pTSA) and joint space width (JSW) preoperatively, postoperatively and at the final follow-up. The FTA was defined as the angle between the anatomical axis of the femur and the tibia in the anteroposterior (AP) view of the knee joint. The MAD was defined as the angle between the center of the ankle joint and the head of femur and that of the knee joint on the weight bearing full-limb AP radiograph. A negative value was given for FTA and MAD in knees in varus alignment. The mPTA was defined as the medial angle between the articular surface and the anatomical axis of the tibia in the AP view. The pTSA was defined as the angle between the tibial plateau and the anatomical axis of the proximal tibia in the lateral view. The JSW was measured with the radiograph taken with the X-ray beam horizontal or parallel to the joint surface and directed at the midpoint or the lower pole of the patella ${ }^{22)}$.

To determine interobserver reliability, 20 cases of randomly selected radiographs that were included in this series were evaluated by two observers (SBJ and LDY). They independently measured preoperative and postoperative displacement values of the radiographs without patient information and repeated the measurements 3 weeks later. Intraobserver and interobserver reliabilities were analyzed using intraclass correlation coefficients (ICCs) with 95\% confidence intervals (CIs).

The extent of cartilage regeneration was assessed by secondlook arthroscopy about 1 year after HTO at the site of eburnation that had been observed arthroscopically on the medial femoral or tibial condyles during removal of plates. The cartilage was classified from grade 0 to grade IV with reference to Outerbridge classification $^{23)}$. Cartilage regeneration was considered to have occurred if a drop in the Outerbridge grade was observed in more than $50 \%$ of the width of the cartilage, and partial cartilage regeneration was diagnosed if a grade change was noted in less than $50 \%$.

\section{Statistical Analysis}

Analysis was performed using IBM SPSS ver. 23.0 (IBM Co., Armonk, NY, USA). Student $t$-test, chi-square test, Fisher exact test, and Mann-Whitney $U$-test were used to show statistical differences between two groups. On demographic data, age, FTA and BMI showed normal distribution, so the Student $t$-test was used, whereas the Mann-whitney $U$-test was used for nonparametric values for the follow-up period. As clinical and radiographic outcomes were nonparametric values, Mann-Whitney $U$-test was used to compare two groups preoperatively, postoperatively and at the final follow-up. Sex and cartilage regeneration were analyzed using Fisher exact and chi-square test, respectively. A p-value $<0.05$ was considered to be statistically significant.

\section{Results}

The clinical outcomes are presented in Table 2. All clinical outcomes were improved in both groups from the postoperative assessment to the final follow-up. All clinical outcomes showed no statistically significant difference between two groups at the final follow-up. The Lysholm knee scoring scale score was statistically

Table 2. Clinical Outcomes of the Two Groups

\begin{tabular}{|c|c|c|c|c|c|}
\hline \multirow{2}{*}{ Group ${ }^{\text {a) }}$} & \multirow{2}{*}{$\begin{array}{c}\text { Pre- } \\
\text { operative }\end{array}$} & \multicolumn{3}{|c|}{ Postoperative } & \multirow{2}{*}{$\begin{array}{c}\text { Final } \\
\text { follow-up }\end{array}$} \\
\hline & & $3 \mathrm{mo}$ & $6 \mathrm{mo}$ & $1 \mathrm{yr}$ & \\
\hline \multicolumn{6}{|l|}{ Lysholm } \\
\hline Group I & 63.3 & 71.8 & 82.4 & 86.4 & 88.3 \\
\hline Group II & 63.4 & 75.2 & 84.2 & 89.2 & 90.1 \\
\hline p-value $e^{b)}$ & 0.417 & 0.041 & 0.141 & 0.914 & 0.217 \\
\hline \multicolumn{6}{|l|}{ VAS } \\
\hline Group I & 6.6 & 6.8 & 4.2 & 3.9 & 3.4 \\
\hline Group II & 6.7 & 7.0 & 4.3 & 3.3 & 3.2 \\
\hline p-value $e^{\text {b) }}$ & 0.924 & 0.829 & 0.610 & 0.040 & 0.691 \\
\hline \multicolumn{6}{|l|}{ HSS } \\
\hline Group I & 66.3 & 71.1 & 80.1 & 83.3 & 85.2 \\
\hline Group II & 66.3 & 72.5 & 82.3 & 86.2 & 87.3 \\
\hline p-value $e^{\text {b) }}$ & 0.845 & 0.448 & 0.056 & 0.008 & 0.267 \\
\hline \multicolumn{6}{|l|}{ WOMAC } \\
\hline Group I & 19.2 & 17.0 & 13.3 & 11.3 & 9.8 \\
\hline Group II & 17.7 & 16.9 & 12.3 & 9.0 & 8.6 \\
\hline p-value ${ }^{b)}$ & 0.689 & 0.575 & 0.957 & 0.603 & 0.631 \\
\hline \multicolumn{6}{|l|}{ Tegner } \\
\hline Group I & 3.1 & 3.4 & 4.4 & 4.7 & 5.1 \\
\hline Group II & 3.5 & 3.6 & 4.3 & 4.9 & 5.3 \\
\hline p-value $e^{b)}$ & 0.237 & 0.732 & 0.834 & 0.665 & 0.472 \\
\hline
\end{tabular}

Values are presented as mean.

Lysholm: Lysholm knee scoring scale, VAS: visual analogue scale score, HSS: Hospital for Special Surgery score, WOMAC: Western Ontario and McMaster Universities score, Tegner: Tegner activity score.

${ }^{a)}$ Group I is kissing group and group II is non-kissing group.

${ }^{b}$ Mann-Whitney $U$-test. 
significantly higher in group II $(\mathrm{p}=0.041)$ on the 3rd postoperative day 3 month postoperatively, but values obtained at the other assessment sessions were not significantly different. The VAS score and the HSS score were statistically significantly higher in group II ( $\mathrm{p}=0.040$ and $\mathrm{p}=0.008) 1$ year postoperatively, but scores obtained at other assessment sessions were not significantly different. Other clinical outcomes did not show statistically significant difference between groups.

Table 3. Radiographic Outcomes of the Two Groups

\begin{tabular}{|c|c|c|c|}
\hline Group $^{\text {a) }}$ & Preoperative & Postoperative & Final follow-up \\
\hline \multicolumn{4}{|l|}{ FTA $\left(^{\circ}\right)$} \\
\hline Group I & Varus 4.6 & Valgus 6.5 & Valgus 6.1 \\
\hline Group II & Varus 3.5 & Valgus 7.4 & Valgus 6.2 \\
\hline p-value ${ }^{b)}$ & 0.366 & 0.157 & 0.926 \\
\hline \multicolumn{4}{|l|}{$\operatorname{MAD}\left({ }^{\circ}\right)$} \\
\hline Group I & Varus 5.7 & Varus 1.3 & Varus 1.5 \\
\hline Group II & Varus 5.2 & Varus 0.9 & Varus 1.1 \\
\hline p-value ${ }^{\text {b) }}$ & 0.577 & 0.471 & 0.366 \\
\hline \multicolumn{4}{|l|}{$\operatorname{mPTA}\left({ }^{\circ}\right)$} \\
\hline Group I & 84.4 & 97.2 & 96.3 \\
\hline Group II & 84.9 & 97.6 & 95.8 \\
\hline p-value ${ }^{b)}$ & 0.917 & 0.886 & 0.835 \\
\hline \multicolumn{4}{|l|}{$\operatorname{pTSA}\left({ }^{\circ}\right)$} \\
\hline Group I & 9.0 & 10.8 & 10.7 \\
\hline Group II & 8.5 & 9.6 & 9.7 \\
\hline p-value ${ }^{\text {b) }}$ & 0.354 & 0.204 & 0.353 \\
\hline \multicolumn{4}{|l|}{ JSW (mm) } \\
\hline Group I & 0.7 & 2.0 & 2.2 \\
\hline Group II & 0.9 & 2.1 & 2.4 \\
\hline p-value ${ }^{\mathrm{b})}$ & 0.277 & 0.353 & 0.389 \\
\hline
\end{tabular}

Values are presented as mean.

FTA: femoral-tibial angle, MAD: mechanical axis deviation, mPTA: medial proximal tibial angle, pTSA: posterior tibial slope angle, JSW: joint space width.

${ }^{a)}$ Group I is kissing group and group II is non-kissing group.

${ }^{b}$ p-values were calculated at the final follow-up using Mann-Whitney $U$-test.
The radiographic outcomes are presented in Table 3. All radiographic outcomes showed no statistically significant difference between two groups at the final follow-up. The mean FTA was corrected by $10.7^{\circ}$ in group I at the final follow-up, and by $9.7^{\circ}$ in group II, and the MAD was corrected by $4.2^{\circ}$ and $4.1^{\circ}$, respectively. But the extent of correction of the FTA and MAD was not statistically significantly different between the preoperative and final follow-up assessments ( $\mathrm{p}=0.926$ and $\mathrm{p}=0.366$ ). The mean correction of mPTA was $11.9^{\circ}$ for group I and $10.9^{\circ}$ for group II at the final follow-up, and that of pTSA was $1.7^{\circ}$ for group I and 1.2 for group II, and mPTA and pTSA showed no statistically significant differences between group ( $\mathrm{p}=0.835$ and $\mathrm{p}=0.353$, respectively). The average correction of JSW was the same with $1.5^{\circ}$ at the final follow-up in two groups, showing no statistically significant intergroup difference $(\mathrm{p}=0.389)$. The ICCs for interobserver and intraobserver reliability were more than 0.8 with the ICCs for FTA and MAD greater than those for the others (Table 4).

Arthroscopic findings obtained during plate removal are described in Table 5. Microfracture resulted in cartilage regeneration in $13(62 \%)$ out of 21 cases in group I and partial cartilage regeneration in 8 cases (38\%) in group I (Fig. 1). It resulted in cartilage regeneration in 19 (86\%) out of 22 cases in group II and partial cartilage regeneration in 3 cases (14\%) in group 2 (Fig. 2). No significant intergroup difference was found $(\mathrm{p}=0.088)$.

Table 5. Outerbridge Classification of Preoperative and Postoperative Arthroscopic Findings

\begin{tabular}{ccccc}
\hline Group & Grade I & Grade II & Grade III & Grade IV \\
\hline Kissing group & & & & \\
Preoperative & 0 & 0 & 0 & 21 \\
Postoperative & 0 & 6 & 15 & 0 \\
Non-kissing group & & & & \\
Preoperative & 0 & 0 & 7 & 15 \\
Postoperative & 0 & 3 & 19 & 0 \\
\hline
\end{tabular}

Values are presented as number of cases.

Table 4. Intraobserver and Interobserver Reliabilities of Radiographic Evaluations

\begin{tabular}{cccccc}
\hline Variable & $\mathrm{FTA}\left({ }^{\circ}\right)$ & $\mathrm{MAD}\left(^{\circ}\right)$ & $\mathrm{mPTA}\left({ }^{\circ}\right)$ & $\mathrm{pTSA}\left({ }^{\circ}\right)$ & $\mathrm{JSW}(\mathrm{mm})$ \\
\hline Intraobserver reliability & $0.975(0.922-0.990)$ & $0.982(0.966-0.995)$ & $0.895(0.718-0.958)$ & $0.825(0.697-0.872)$ & $0.873(0.667-0.951)$ \\
Interobserver reliability & $0.940(0.780-0.984)$ & $0.939(0.834-0.975)$ & $0.808(0.494-0.925)$ & $0.836(0.690-0.883)$ & $0.853(0.460-0.955)$ \\
\hline
\end{tabular}

Values are intraclass correlation coefficients (95\% confidence intervals).

FTA: femoral-tibial angle, MAD: mechanical axis deviation, mPTA: medial proximal tibial angle, pTSA: posterior tibial slope angle, JSW: joint space width. 

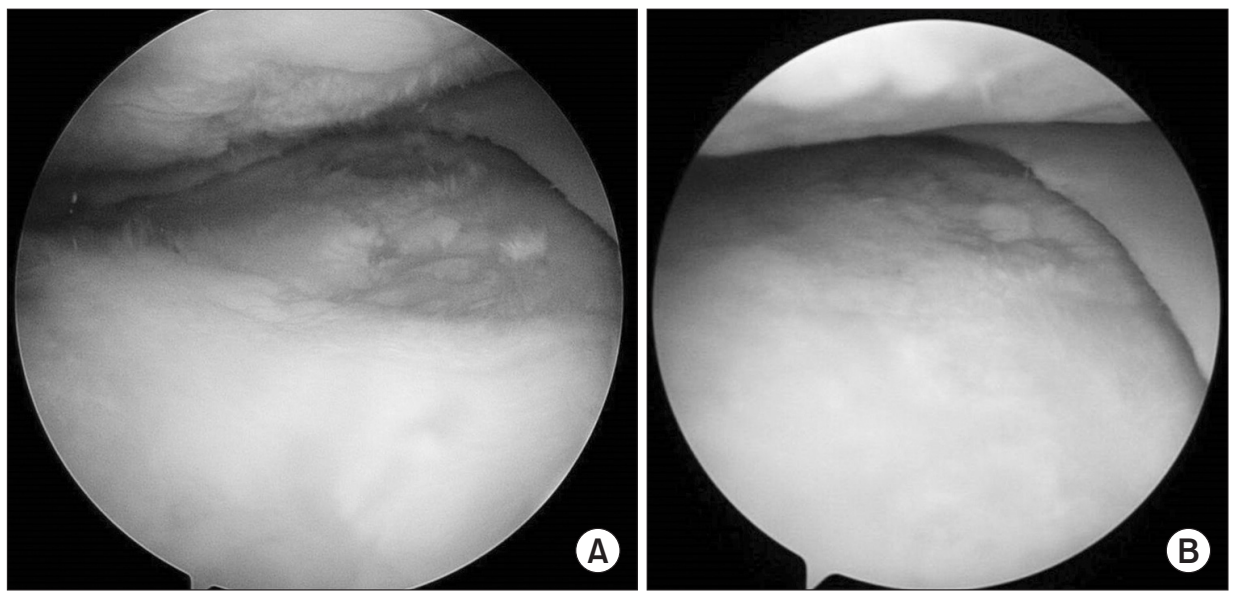

Fig. 1. A 62-year-old female with a kissing lesion. (A) Initial arthroscopy showed a cartilage defect involving both the medial femoral condyle and medial tibial condyle. (B) After high tibial osteotomy and microfracture, cartilage was regenerated.
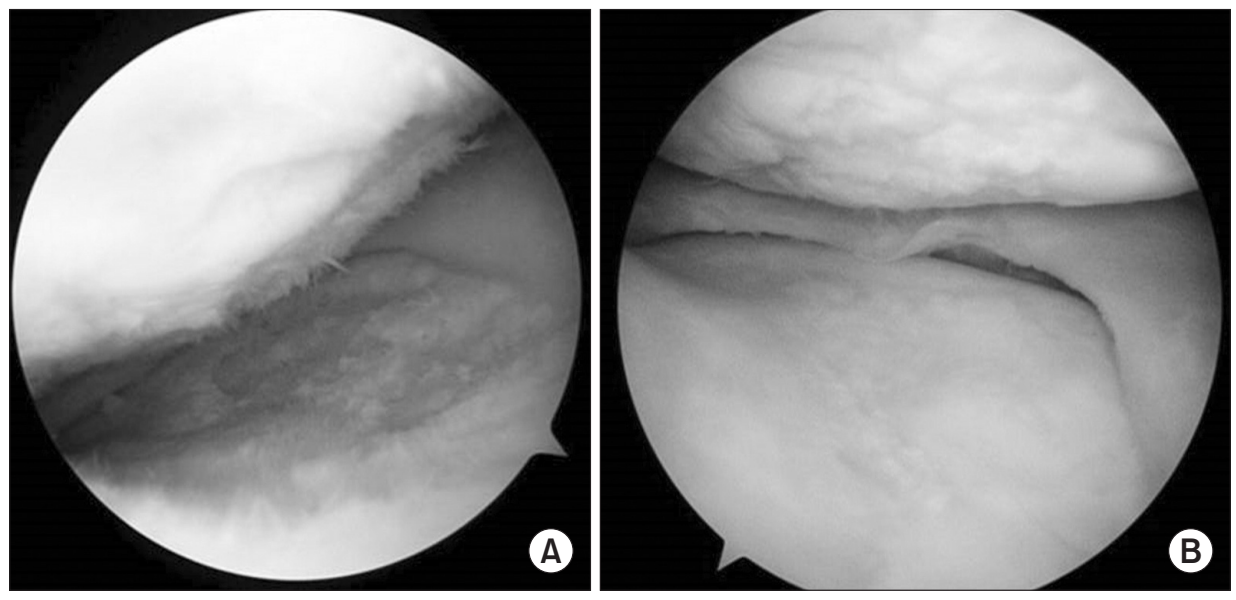

Fig. 2. A 59-year-old female with a nonkissing lesion. (A) Initial arthroscopy showed a cartilage defect involving both the medial femoral condyle and medial tibial condyle. (B) After high tibial osteotomy and microfracture, cartilage was regenerated.

\section{Discussion}

With the increase of geriatric diseases, the prevalence of medial knee arthritis is on the rise $e^{24)}$. Unicompartmental arthritis of the knee can be found in diverse age groups and treated with surgical methods such as HTO and UKA. Since the two methods are comparably effective in treating knee arthritis, the patient's preference is important in deciding a surgical method. Above all, however, it is important to consider the factors that can influence the postoperative survival rate including body mass index, severity of varus deformity, correction angle and severity of arthritis. Furthermore, appropriate surgical techniques and good rehabilitation are also required ${ }^{25}$.

While both HTO and UKA can be ideally applied to most patients with medial knee arthritis, appropriate surgical techniques and comparative efficacy of the two procedures have been the subjects of controversy for borderline patients. HTO was once known as a relative contradiction for patients with advanced arthritis accompanied by a kissing lesion and knee arthroplasty was considered to be effective in most cases. However, there are many cases where knee arthroplasty can hardly be applied due to the risk of infection, difficulty in management and young age. In addition, extended life expectancy has made patients more unwilling to undergo revision arthroplasty, and long-term followup reports have shown that UKA is not effective in all cases ${ }^{13,26,27}$. Borderline patients when it comes to the application of HTO and UKA are increasing. Some authors reported that HTO would be more helpful in the long-term than UKA for relatively young patients with severe arthritis ${ }^{28)}$. Our study also showed that although the follow-up was short, there was no significant intergroup difference in the Lysholm score, VAS score, HSS score, WOMAC score, Tegner activity score, radiographic findings, and cartilage regeneration.

UKA was a more common surgical method for patients with advanced arthritis accompanied by a kissing lesion than HTO. However, HTO needs to be performed in the presence of deformity, malalignment and relatively less advanced patellofemoral arthritis. In addition, it is difficult to perform UKA in younger 
patients even in the presence of an advanced degenerative change of the knee joint accompanied by hypertrophy of the anterior cruciate ligament ${ }^{25-27)}$. In such cases, the treatment goal is to slow down the disease progress and HTO can delay an arthroplasty.

Our findings showed that HTO could be applicable to patients who had advanced arthritis with relatively less severe patellofemoral arthritis and mild meniscus damage that can be treated with partial meniscectomy or suture. As outcomes of HTO were not bad in the short-term follow-up, it can be expected to not only significantly delay the use of UKA or TKA for young patients but also reduce the number of reoperations. Therefore, HTO can be more satisfactory in terms of the survival rate than arthroplasty $^{25)}$. It may be difficult to conclude that HTO has a good survival rate because of the short-term follow-up. However, our findings suggest that HTO can be a good alternative treatment even in the case of medial compartment arthritis with a kissing lesion. Preoperative efforts to select appropriate patients and analyze associated factors can be helpful for adequate preoperative planning and improvement of postoperative survival rate. While it is necessary to conduct a long-term study on HTO to validate our results, we suggest that research on new indications for HTO should also be conducted.

Some authors reported poor results of microfracture when the procedure was performed on the medial femoral condyle in the weight bearing area, which was greater than $2 \mathrm{~cm}^{2}$, or in the presence of osteoarthritis ${ }^{29)}$. We also question how microfracture resulted in satisfactory clinical outcomes despite the big size of cartilage defect, which should be elucidated in further research.

There have been some recent reports on the outcomes of HTO with bone marrow stimulation in Outerbridge classification III or IV cartilage defect patients. Although there is some controversy on the use of HTO with bone marrow stimulation ${ }^{30)}$, some authors reported favorable outcomes of HTO with microfracture in Outerbridge grade III and IV chondropathies on the medial femoral and tibial joint surface that can be diagnosed as a kissing lesion as in our study ${ }^{14)}$. Our results were congruent with the study, showing satisfactory outcomes in the kissing lesion group.

The kissing lesion and non-kissing lesion groups had different Outerbridge stages preoperatively. Arthroscopy revealed improvement in both groups although lower Outerbridge stages were observed in the non-kissing group in the second look arthroscopy. However, we think that the significance of this study is that the clinical and radiographic outcomes showed no remarkable differences between the two groups, and cartilage regeneration was observed in both groups.

This study has some limitations. The short-term follow-up was not sufficient for survival rate analysis; therefore, it is necessary to conduct a prospective, constant follow-up. Our results may not be was replicated with closed wedge osteotomy because we performed only open wedge osteotomy in all cases. In addition, there was no control group where HTO alone without microfracture was performed for the kissing lesion. Last, we think that the clinical outcomes were better than those in other studies in part because the patients in our study were younger.

\section{Conclusions}

HTO provided relatively satisfactory clinical and radiographic outcomes in young patients with a kissing lesion accompanied by relatively mild meniscus damage in the short term follow-up. HTO might be a good alternative treatment option for medial compartment arthritis with a kissing lesion in relatively young patients.

\section{Conflict of Interest}

No potential conflict of interest relevant to this article was reported.

\section{References}

1. Jackson JP, Waugh W. Tibial Osteotomy for Osteoarthritis of the Knee. Proc R Soc Med. 1960;53:888.

2. Svard UC, Price AJ. Oxford medial unicompartmental knee arthroplasty: a survival analysis of an independent series. J Bone Joint Surg Br. 2001;83:191-4.

3. Insall JN, Joseph DM, Msika C. High tibial osteotomy for varus gonarthrosis: a long-term follow-up study. J Bone Joint Surg Am. 1984;66:1040-8.

4. Naudie D, Bourne RB, Rorabeck CH, Bourne TJ. The Install Award: survivorship of the high tibial valgus osteotomy: a 10- to -22-year followup study. Clin Orthop Relat Res. 1999; (367):18-27.

5. Preston S, Howard J, Naudie D, Somerville L, McAuley J. Total knee arthroplasty after high tibial osteotomy: no differences between medial and lateral osteotomy approaches. Clin Orthop Relat Res. 2014;472:105-10.

6. Naudie DD, Amendola A, Fowler PJ. Opening wedge high tibial osteotomy for symptomatic hyperextension-varus thrust. Am J Sports Med. 2004;32:60-70.

7. Getgood A, Collins B, Slynarski K, Kurowska E, Parker D, Engebretsen L, MacDonald PB, Litchfield R. Short-term 
safety and efficacy of a novel high tibial osteotomy system: a case controlled study. Knee Surg Sports Traumatol Arthrosc. 2013;21:260-9.

8. Harris JD, McNeilan R, Siston RA, Flanigan DC. Survival and clinical outcome of isolated high tibial osteotomy and combined biological knee reconstruction. Knee. 2013;20: 154-61.

9. Rudan JF, Simurda MA. High tibial osteotomy: a prospective clinical and roentgenographic review. Clin Orthop Relat Res. 1990;(255):251-6.

10. Flecher X, Parratte S, Aubaniac JM, Argenson JN. A 12-28year followup study of closing wedge high tibial osteotomy. Clin Orthop Relat Res. 2006;452:91-6.

11. Ivarsson I, Myrnerts R, Gillquist J. High tibial osteotomy for medial osteoarthritis of the knee. A 5 to 7 and 11 year follow-up. J Bone Joint Surg Br. 1990;72:238-44.

12. Ekhtiari S, Haldane CE, de Sa D, Simunovic N, Musahl V, Ayeni OR. Return to work and sport following high tibial osteotomy: a systematic review. J Bone Joint Surg Am. 2016;98: 1568-77.

13. W-Dahl A, Robertsson O, Lidgren L. Surgery for knee osteoarthritis in younger patients. Acta Orthop. 2010;81:161-4.

14. Pascale W, Luraghi S, Perico L, Pascale V. Do microfractures improve high tibial osteotomy outcome? Orthopedics. 2011; 34:e251-5.

15. van den Bekerom MP, Patt TW, Rutten S, Raven EE, van de Vis HM, Albers GH. Arthroscopic debridement for grade III and IV chondromalacia of the knee in patients older than 60 years. J Knee Surg. 2007;20:271-6.

16. Kellgren JH, Lawrence JS. Radiological assessment of osteoarthrosis. Ann Rheum Dis. 1957;16:494-502.

17. Lysholm J, Gillquist J. Evaluation of knee ligament surgery results with special emphasis on use of a scoring scale. Am J Sports Med. 1982;10:150-4.

18. Carlsson AM. Assessment of chronic pain: I. Aspects of the reliability and validity of the visual analogue scale. Pain. 1983;16:87-101.

19. Insall JN, Dorr LD, Scott RD, Scott WN. Rationale of the Knee Society clinical rating system. Clin Orthop Relat Res.
1989;(248):13-4.

20. Bellamy N, Buchanan WW, Goldsmith CH, Campbell J, Stitt LW. Validation study of WOMAC: a health status instrument for measuring clinically important patient relevant outcomes to antirheumatic drug therapy in patients with osteoarthritis of the hip or knee. J Rheumatol. 1988;15:1833-40.

21. Tegner Y, Lysholm J. Rating systems in the evaluation of knee ligament injuries. Clin Orthop Relat Res. 1985;(198):43-9.

22. Ravaud P, Chastang C, Auleley GR, Giraudeau B, Royant V, Amor B, Genant HK, Dougados M. Assessment of joint space width in patients with osteoarthritis of the knee: a comparison of 4 measuring instruments. J Rheumatol. 1996; 23:1749-55.

23. Outerbridge RE. The etiology of chondromalacia patellae. J Bone Joint Surg Br. 1961;43:752-7.

24. Dillon CF, Rasch EK, Gu Q, Hirsch R. Prevalence of knee osteoarthritis in the United States: arthritis data from the Third National Health and Nutrition Examination Survey 1991-94. J Rheumatol. 2006;33:2271-9.

25. Xing Z, Katz J, Jiranek W. Unicompartmental knee arthroplasty: factors influencing the outcome. J Knee Surg. 2012; 25:369-73.

26. Warme BA, Aalderink $\mathrm{K}$, Amendola A. Is there a role for high tibial osteotomies in the athlete? Sports Health. 2011;3: 59-69.

27. Mancuso F, Dodd CA, Murray DW, Pandit H. Medial unicompartmental knee arthroplasty in the ACL-deficient knee. J Orthop Traumatol. 2016;17:267-75.

28. Miller BS, Downie B, McDonough EB, Wojtys EM. Complications after medial opening wedge high tibial osteotomy. Arthroscopy. 2009;25:639-46.

29. Alparslan L, Winalski CS, Boutin RD, Minas T. Postoperative magnetic resonance imaging of articular cartilage repair. Semin Musculoskelet Radiol. 2001;5:345-63.

30. Akizuki S, Yasukawa Y, Takizawa T. Does arthroscopic abrasion arthroplasty promote cartilage regeneration in osteoarthritic knees with eburnation? A prospective study of high tibial osteotomy with abrasion arthroplasty versus high tibial osteotomy alone. Arthroscopy. 1997;13:9-17. 